\title{
Optimizing the CRISPR/Cas9 system for genome editing in grape by using grape promoters
}

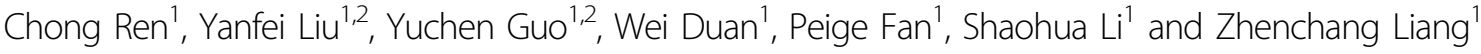

\begin{abstract}
The efficacy of the CRISPR/Cas9 system in grapevine (Vitis vinifera L.) has been documented, but the optimization of this system, as well as CRISPR/Cas9-mediated multiplex genome editing, has not been explored in this species. Herein, we identified four VVU3 and VVU6 promoters and two ubiquitin (UBQ) promoters in grapevine and demonstrated that the use of the identified VVU3/U6 and UBQ2 promoters could significantly increase the editing efficiency in grape by improving the expression of sgRNA and Cas9, respectively. Furthermore, we conducted multiplex genome editing using the optimized CRISPR/Cas9 vector that contained the conventional multiple sgRNA expression cassettes or the polycistronic tRNA-sgRNA cassette (PTG) by targeting the sugar-related tonoplastic monosaccharide transporter (TMT) family members TMT1 and TMT2, and the overall editing efficiencies were higher than 10\%. The simultaneous editing of TMT1 and TMT2 resulted in reduced sugar levels, which indicated the role of these two genes in sugar accumulation in grapes. Moreover, the activities of the $\mathrm{VvU} 3, \mathrm{VV} U 6$, and $\mathrm{UBQ} 2$ promoters in tobacco genome editing were demonstrated by editing the phytoene desaturase (PDS) gene in Nicotiana benthamiana leaves. Our study provides materials for the optimization of the CRISPR/Cas9 system. To our knowledge, our simultaneous editing of the grape TMT family genes TMT1 and TMT2 constitutes the first example of multiplex genome editing in grape. The multiplex editing systems described in this manuscript expand the toolbox of grape genome editing, which would facilitate basic research and molecular breeding in grapevine.
\end{abstract}

\section{Introduction}

The advent of the clustered regularly interspaced short palindromic repeats (CRISPR)/CRISPR-associated protein 9 (Cas9) system has been revolutionizing genome editing and genetic therapy ${ }^{1,2}$. In recent years, CRISPR/Cas9 has been more rapidly and widely applied in numerous plant species due to its simplicity, high efficiency, and versatility compared with previous genome editing technologies ${ }^{3-5}$. The most popular CRISPR/Cas9 system was initially derived from the adaptive immune system in Streptococcus pyogenes ${ }^{6}$, and an engineered system in which a single guide RNA (sgRNA) and the Cas9 nuclease are needed for genome editing ${ }^{1}$. The Cas9 nuclease can

\footnotetext{
Correspondence: Zhenchang Liang (z1249@ibcas.ac.cn)

${ }^{1}$ Beijing Key Laboratory of Grape Science and Enology, and CAS Key Laboratory of Plant Resources, Institute of Botany, The Innovative Academy of Seed Design, The Chinese Academy of Sciences, 100093 Beijing, People's Republic of China

${ }^{2}$ University of Chinese Academy of Sciences, 100049 Beijing, People's Republic of China
}

produce double-stranded DNA breaks, whereas the sgRNA specifically directs the Cas9 protein to its complementary DNA target site through RNA-DNA base pairing ${ }^{1}$. The expression of sgRNA and Cas 9 has been shown to influence the editing efficiency of the CRISPR/ Cas9 system $^{7-10}$.

In the CRISPR/Cas9 system, the expression of Cas 9 is generally driven by an RNA polymerase II (Pol II) promoter. During the early applications of the CRISPR/Cas9 system in plants, the CaMV35S promoter was often used to express the Cas 9 gene ${ }^{10-12}$. Recently, plant ubiquitin (UBQ) promoters have been isolated and employed in the CRISPR/ Cas9 system in place of the 35 S promoter ${ }^{13-15}$. The Pol III promoters of small nuclear RNA (snRNA) genes, such as U3 and U6, are commonly used to drive sgRNA expression in plants and animals ${ }^{16,17}$. U6 snRNAs participate in the intron splicing of pre-mRNA in the nucleus ${ }^{18}$, whereas U3 snRNAs are involved in pre-rRNA processing ${ }^{19,20}$. Unlike other Pol III-transcribed genes that contain 
intragenic promoter elements, U6 snRNA genes only possess a conserved TATA box and the upstream sequence element (USE) in their promoters ${ }^{18,21}$. In contrast, the promoter structures of U3 snRNA genes vary among different species, particularly metazoans ${ }^{20}$. In higher plants, however, a canonical TATA-like box and well-conserved proximal USE are present in the U3 promoters ${ }^{20,21}$. Both of these promoters are capable of producing high levels of sgRNA, which typically have a length of $\sim 200$ nucleotides ${ }^{22}$. In general, the Arabidopsis U6 (AtU6) and AtU3 promoters are extensively used in CRISPR/Cas9 vectors for genome editing in dicot plants, whereas the rice (Oryza sativa) U6 (OsU6) and OsU3 promoters are primarily applied in genome engineering in monocot plants ${ }^{16,23}$. Many CRISPR/ Cas9 toolboxes have been developed and optimized based on the Pol III promoters isolated from the two model plant species ${ }^{16,21,23}$. Recently, plant species-specific U6 promoters for CRISPR/Cas9-mediated genome editing were adopted in soybean ${ }^{8}, \operatorname{cotton}^{10,24}$, apple ${ }^{25}$, and wheat ${ }^{26}$, and these studies revealed that the use of plant species-specific Pol III promoters could contribute to increased sgRNA levels and thus, result in enhanced editing efficiencies ${ }^{8-10}$. However, the identification and application of plant species-specific Pol III promoters have not been fully explored, and U3 and U6 promoters in other plant species are expected to be characterized during the development and optimization of CRISPR/Cas9 systems for these species. As a fruit crop that is widely cultivated worldwide, grape (Vitis ssp.) is economically important. CRISPR/Cas9-mediated genome editing in grape (Vitis vinifera L.) was first reported in 2016 (ref. ${ }^{27}$ ), and since then, the AtU6 or AtU3 promoters have been used to drive the expression of sgRNAs during grape genome editing ${ }^{27-31}$. Nonetheless, the grape Pol III promoter has not yet been identified.

Multiplex genome editing can be achieved by stacking multiple sgRNAs in a single CRISPR vector ${ }^{13}$. The simplest and most common approach involves the use of multiple U3 and/or U6 promoters for the expression of different $\operatorname{sgRNAs}{ }^{32}$. Additionally, approaches based on self-cleaving ribozymes (RZ), tRNA, or Csy4 have also been used to produce multiple sgRNAs ${ }^{33,34}$. Multiplex genome editing, which enables the simultaneous targeting of several related or unrelated genes, has been applied in many plant species ${ }^{35}$. In this method, different alleles of the same gene, homoeoalleles in polyploid plants, or even a gene family could be simultaneously edited through multiplex gene editing ${ }^{35,36}$. The greatest potential of multiplex genome editing has been shown by editing different genes involved in the control of distinct traits in plants. For instance, the CRISPR/Cas9-mediated targeting of multiple genes that control the time of flowering and plant morphology can accelerate the domestication of wild tomato or directly develop customized tomato for urban agriculture ${ }^{37,38}$. In recent years, an increasing number of reported studies have implemented targeted genome editing in grape, and almost all the studies were performed with one or several sgRNAs targeting single genes $^{27-31,39}$. The simultaneous editing of different genes of interest could improve different traits of grapes, which would be extremely significant for shortening the period of grape breeding. However, CRISPR/Cas9-mediated multiplex genome editing involving different genes has not been explored in grape.

In the present study, we first identified four $\mathrm{VvU} 3$ and VvU6 promoters and two UBQ promoters in grape and investigated their efficacy and efficiency in genome editing by targeting the grape phytoene desaturase $(P D S)$ gene. The use of grape VvU3/U6 and UBQ2 promoters can clearly improve the editing efficiencies in grape cells and stable transgenic plants by increasing the expression of sgRNA and Cas9, respectively. We then optimized the CRISPR/Cas9 system using the VvU6 and UBQ2 promoters instead of the AtU6 and $35 \mathrm{~S}$ promoters, respectively, and developed a multiplex genome editing system containing the traditional multiple sgRNA (multi-sgRNA) expression cassettes or the polycistronic tRNA-sgRNA cassette (PTG). The simultaneous editing of the grape tonoplastic monosaccharide transporter (TMT) genes TMT1 and TMT2 demonstrated the efficacy of the two systems. Additionally, the successful editing of the tobacco NbPDS gene suggests that the identified VvU3 and VvU6 promoters, as well as the UBQ2 promoter, might be applied in other dicot plants.

\section{Results}

Identification of U3, U6, and UBQ promoters in grapevine

In previous studies on genome editing in grape, AtU6 and $35 \mathrm{~S}$ promoters were commonly used to drive the expression of sgRNAs and Cas9, respectively ${ }^{27,29,31,40}$. In an effort to optimize the CRISPR/Cas9 system in grape, we conducted BLAST searches of the grape genome $(V$. vinifera L.) using the Arabidopsis AtU6-26 and AtU3b genes as the queries, and identified four $V v U 6$ and $V v U 3$ snRNA genes showing high sequence similarities to the queries (Table 1). The two VvU6 (VvU6.1 and VvU6.2) and two $V v U 3$ ( $V v U 3.1$ and $V v U 3.2)$ snRNA genes exhibited relatively conserved snRNA transcript sequences compared with the corresponding AtU6 and AtU3 genes. However, the promoter regions of these genes were divergent between the two species, with the exception of the USE and TATA-like box, which are required for transcription (Fig. 1a). The presence of the USE and TATA-like box in the promoters of the $V v U 3$ and $V v U 6$ genes suggests that these Pol III promoters might be involved in effective transcription. We therefore cloned the promoter regions upstream of the transcription initiation site of the four $V v U 3$ and $V v U 6$ genes as the VvU3 and VvU6 promoters for subsequent experiments 
(Table 1 and Supplementary Fig. S1). In addition, we screened two grape $U B Q$ genes that are constitutively expressed in grapevine (Table 1 and Supplementary Fig. S2). In this study, 748-bp and 845-bp DNA fragments upstream of the start codon "ATG" were amplified as putative UBQ promoters (Supplementary Fig. S3).

\section{Assay of the activities of the $\mathrm{VvU3}, \mathrm{VvU6}$, and UBQ promoters via transient expression in tobacco and grapevine leaves}

To investigate whether the amplified VvU3 and VvU6 promoters could drive the expression of sgRNA, we used these Pol III promoters instead of the AtU6 promoter in the pCACRISPR/Cas9 vector $^{27}$ containing a

Table 1 Amplified Pol II and Pol III promoters in grape.

\begin{tabular}{lll}
\hline Promoter & Gene ID & Size (bp) \\
\hline VVU3.1 & ENSRNA049469468 & 393 \\
VVU3.2 & ENSRNA049467827 & 483 \\
VVU6.1 & ENSRNA049469148 & 425 \\
VVU6.2 & ENSRNA049469157 & 591 \\
UBQ1 & VIT_19s0177 g00040 & 748 \\
UBQ2 & VIT_19s0177 g00070 & 845 \\
\hline
\end{tabular}

sgRNA targeting the grape PDS gene. The CRISPR vectors were then introduced into Nicotiana benthamiana leaves via Agrobacterium-mediated infiltration for transient expression. The expression of $P D S$ sgRNA was inspected by quantitative real-time PCR (qRT-PCR). According to the results, the VvU3 and VvU6 promoters successfully promoted the expression of PDS sgRNA in tobacco leaves, and the sgRNA expression levels driven by the VvU3 and VvU6 promoters were comparable or higher than those coordinated by the AtU6 promoter (Fig. 2a).

Similarly, the activities of the UBQ promoters were first assessed in tobacco leaves, and the $\beta$-glucuronidase (GUS) gene fused to the UBQ promoters was used as the reporter. As shown in Fig. 2b, both UBQ1 and UBQ2 promoted the expression of the GUS gene in tobacco leaves, and the activity of UBQ2 was higher than that of UBQ1, as indicated by the finding that the leaf region infiltrated with the UBQ2:GUS construct exhibited stronger GUS staining intensity compared with the region transformed with the UBQ1:GUS vector (Fig. 2b). However, no GUS staining was observed in the grapevine leaves after infiltration with the UBQ1: GUS construct, whereas strong GUS staining was observed with the UBQ2 promoter (Fig. 2c and Supplementary Fig. S4). Based on these results, the UBQ2 promoter was selected for further study.

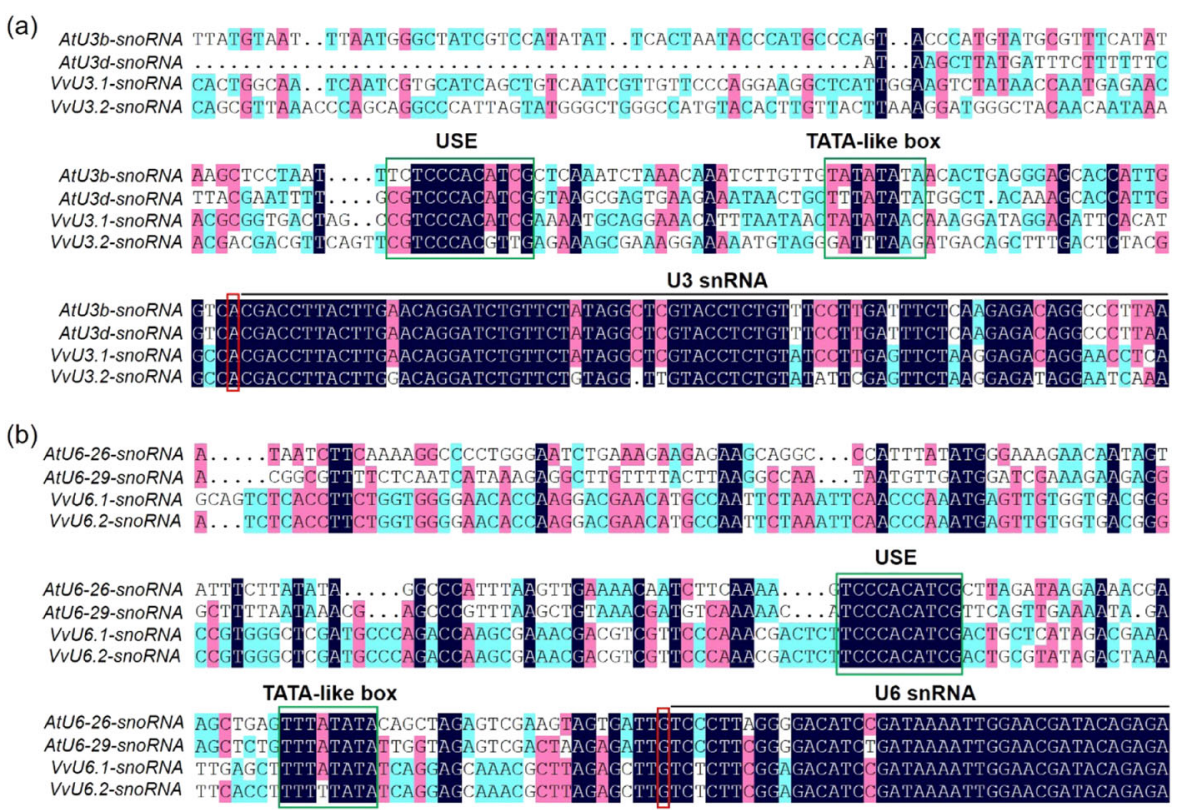

Fig. 1 Analysis of grape VvU3 and VvU6 promoter sequences. Multiple alignments of the grape and Arabidopsis U3 (a) and U6 (b) snRNA genes are shown. Black lines indicate snRNA transcripts. The conserved elements USE (upstream sequence element) and TATA-like box are indicated by green boxes. The nucleotides " $A$ " (adenine) and " $G$ " (guanine) recognized by the U3 and U6 promoters for transcription initiation are labeled in red boxes. Different colors denote different levels of sequence identity. The nucleotides with $100 \%$ identity are highlighted in black, and those with $\geq 75 \%$ and $\geq 50 \%$ identity are highlighted in red and blue, respectively. The multiple sequence alignments were performed using DNAMAN software 


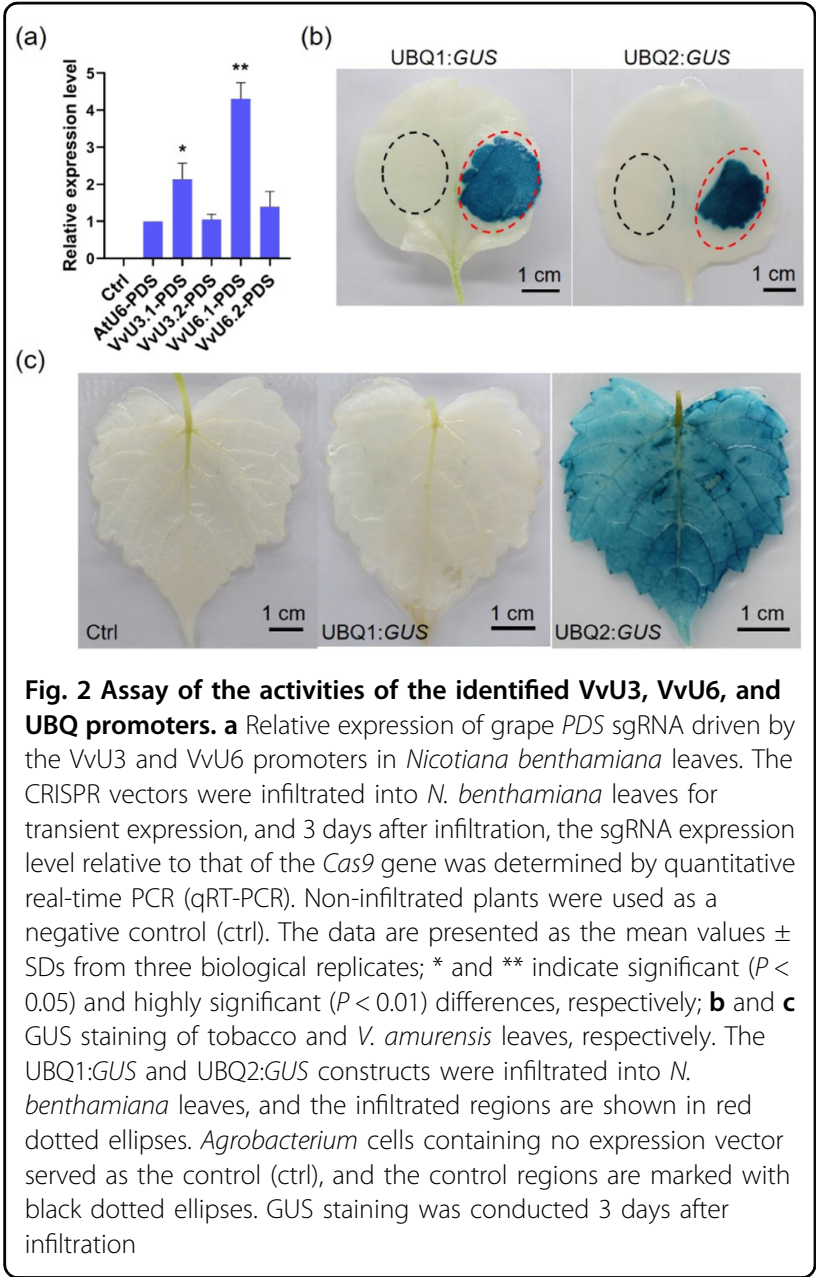

\section{The use of the VvU3/U6 and UBQ2 promoters contributes to genome editing in grape}

It has been reported that using plant species-specific promoters could increase the expression of sgRNA/Cas9, resulting in enhanced editing efficiencies ${ }^{8-10}$. To determine whether the use of grape $\mathrm{VvU} 3 / \mathrm{U} 6$ and $\mathrm{UBQ} 2$ promoters results in improved genome editing efficiency in grape, we constructed optimized CRISPR/Cas9 vectors based on the backbone of the pCAMBIA2300 vector using the grape UBQ2 promoter to drive the expression of Cas 9 and the VvU3/U6 promoters to express PDS sgRNA (Fig. 3a). The optimized CRISPR vectors were designated pCA-UBQ2Cas9-VvU3/U6-PDS and introduced into embryogenic cells derived from 41B grapevine rootstock via Agrobacteriummediated transformation, and kanamycin-resistant cells were obtained after antibiotic-dependent selection (Fig. 3b). Exogenous T-DNA insertions were identified by PCR using Cas9-specific primers (Supplementary Table S1). The results revealed that the kanamycin-resistant cells contained the exogenous Cas9 gene (Fig. 3c), which suggested that the CRISPR vectors had been successfully introduced into the grape cells. We therefore checked the target site of the PDS gene in these transgenic cells. Given that the presence of unedited (or wild-type) sequences from untransformed cells could influence the identification of mutated sequences, we employed the restriction enzyme (RE)/PCR approach for the detection of mutagenesis in the PDS gene. The isolated genomic DNA (gDNA) from the grape cells was treated with the SspI restriction enzyme to digest the wild-type sequences, and the mutated DNA sequences, which are recalcitrant to enzyme digestion, could be enriched via subsequent PCR amplification (Fig. 3d). The desired bands were purified and sent for Sanger sequencing. As expected, indel (deletions or insertions) mutations at the target site were observed in the VvU3-PDS and VvU6-PDS transgenic cells (Fig. 3e). Most of the mutations were short nucleotide $(<10 \mathrm{bp})$ insertions or deletions (Fig. 3e), which is consistent with our previous results obtained using the AtU6 promoter $^{40}$. In addition, mutations consisting of $\geq 18$-bp deletions were also observed in the VvU3.1-PDS and VvU6.1PDS transgenic cells (Fig. 3e). These results demonstrated the efficacy of the $\mathrm{VvU} 3, \mathrm{VvU6}$, and UBQ2 promoters in CRISPR/Cas9-mediated genome editing in grape.

The expression of sgRNA and Cas9 in transgenic grape cells was measured by qRT-PCR, and the results showed that the transcript levels of the PDS sgRNA driven by the $\mathrm{VvU} 3$ and VvU6 promoters were higher ( $>3$-fold) than those obtained using the AtU6 promoter (Fig. 4a). In addition to sgRNA expression, the transcript abundance of Cas 9 was significantly increased when using the UBQ2 promoter (Fig. 4a). The editing efficiencies in the transgenic cells were assessed using the restriction fragment length polymorphism (RFLP) method. The target fragment of the PDS gene was amplified from the AtU6-, VvU3.1-, VvU3.2-, VvU6.1-, and VvU6.2-PDS transgenic cells by PCR, and the PCR products were digested with the $S s p$ I enzyme. As mentioned above, successful targeted editing destroyed the $S s p$ I restriction enzyme site, resulting in accumulation of the mutated sequences. The indel frequency can be calculated according to the intensity of undigested PCR bands ${ }^{40,41}$. The RFLP analysis showed that the use of the VvU3 and VvU6 promoters resulted in higher editing efficiencies, and the indel frequencies ranged from $14.65 \%$ to $22.10 \%$ (Fig. $4 \mathrm{~b}$ and Supplementary Table S2). In contrast, the editing efficiency obtained with the AtU6 promoter was 13.67\% (Fig. 4b and Supplementary Table S2).

Interestingly, the editing efficiencies with the VvU3 promoters were slightly lower than those obtained with the VvU6 promoters (Fig. 4b). One possible reason is that the eukaryotic U3 promoter requires adenine (A) for transcription initiation, whereas the U6 promoter recognizes guanine $(G)$ to initiate transcription. The additional "A" or "G" at the 5 ' ends of the mature sgRNA could affect the editing efficiencies ${ }^{42,43}$. Therefore, we adopted VvU6PDS transgenic cells for plant regeneration, and albino 
(a)

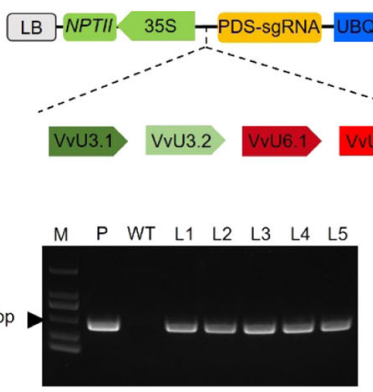

(b)

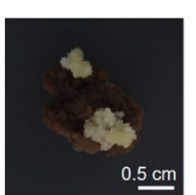

(d)

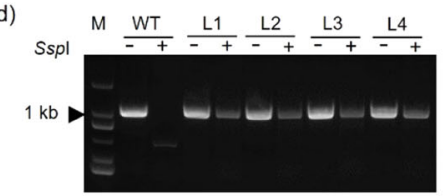

(e)

Sspl

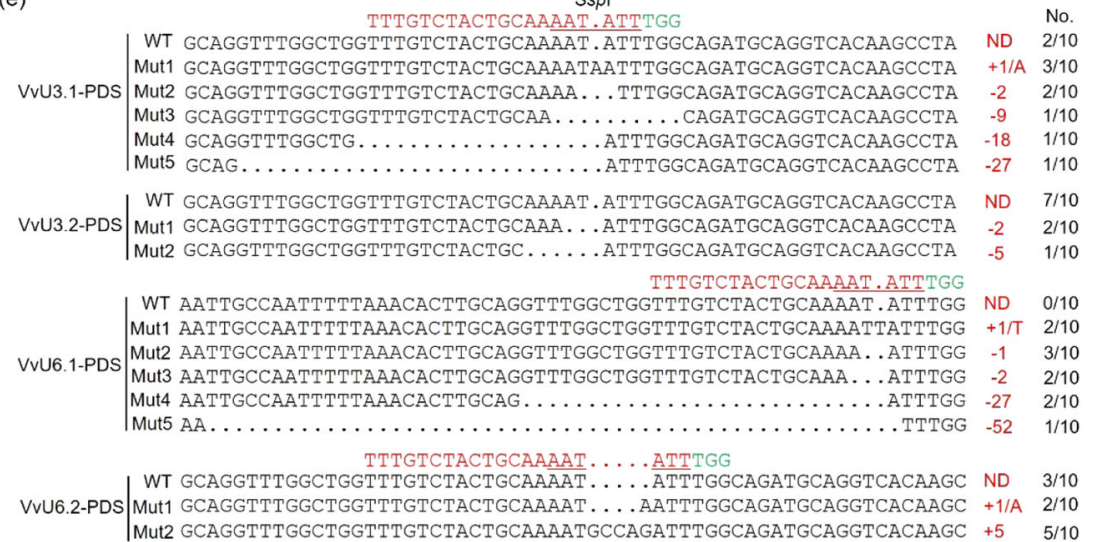

Fig. 3 Targeted mutations generated using the VvU3, VvU6, and UBQ2 promoters. a Schematic illustration of sgRNA/Cas9 vectors. The cloned VVU3 and VVU6 promoters were used instead of the AtU6 promoter, whereas the CaMV35S promoter that drives the expression of Streptococcus pyogenes Cas9 (SpCas9) was replaced by the UBQ2 promoter. NPTII, neomycin phosphotransferase gene; $T_{N O S}$, terminator of nopaline synthase gene; $L B$, left border; $R B$, right border. b Resistant $41 B$ cells generated on selective medium. c PCR identification of exogenous T-DNA insertions. Cas9specific primers were used to identify the T-DNA in 41B cells. The CRISPR vector and wild-type (WT) cells were used as positive (P) and negative controls, respectively. Lanes 1-5 (L1-5) represent samples from VvU3.1-PDS-, VvU3.2-PDS-, VvU6.1-PDS-, VvU6.2-PDS-, and AtU6-PDS-containing cells, respectively. M, DNA marker. $\mathbf{d}$ Restriction (RE)/PCR assay. The target sequence of the grape PDS gene contained an Sspl enzyme site, genomic DNA (gDNA) prepared from VvU3.1-PDS- (L1), VvU3.2-PDS- (L2), VvU6.1-PDS- (L3), and VvU6.2-PDS-containing (L4) cells was treated with Sspl, and mutated sequences were enriched by PCR due to the disruption of the enzyme site after editing. The WT gDNA was used as a negative control. +, Sspl digestion; -, no digestion. e Sequencing results of the target sequences. The enriched target sequences after RE/PCR shown in $\mathbf{d}$ were cloned into the pLB vector for Sanger sequencing. For each sample, a total of 10 clones were sequenced. The mutation type and corresponding number of clones are shown in red and black on the right, respectively. ND, not detected. WT, wild-type sequences. Mut, mutated sequences. The PAM sequence is indicated in green, and the Sspl enzyme site is underlined

plants were obtained as expected (Fig. 4c). According to the results, the mutation rates of VvU6.1-PDS (34.48\%, $10 / 29)$ and VvU6.2-PDS $(43.24 \%, 16 / 37)$ were higher than that of AtU6-PDS $(23.53 \%, 4 / 17)$, and homologous or biallelic plants were obtained with the VvU6.1 and VvU6.2 promoters at rates of $13.79 \%$ and $16.22 \%$, respectively (Fig. 4d and Supplementary Fig. S5). Collectively, these results suggested that the use of speciesspecific VvU3/U6 and UBQ2 promoters resulted in high editing efficiency in grapes.

\section{High-efficiency multiplex genome editing in grape using grape promoters}

Given that the use of VvU3/VvU6 and UBQ2 promoters resulted in efficient genome editing, we hypothesized that these promoters can be used to express different sgRNAs for multiplex genome editing in grape. Multiplex genome editing enables researchers to study the functions of several related genes or even perform metabolic engineering in plants ${ }^{36,44}$. However, previous studies on genome editing in grape have primarily focused on single genes $^{27-31,39}$, and editing studies involving different genes of interest have not been reported. To investigate the efficacy of the CRISPR/Cas9-based multiplex editing system in grape, we edited the TMT gene family in 41B grape cells, which have been successfully used to explore sugar uptake and accumulation ${ }^{45}$. Grape has three TMT genes, namely TMT1 (VIT_18s0122g00850), TMT2 (VIT_03s0038g03940), and TMT3 (VIT_07s0031g02270), which show high similarity to their homologs in Arabidopsis $^{46}$. However, the role of these three genes in sugar accumulation in grape has not been explored. By investigating the expression profiles of the three genes in different tissues or organs at different developmental stages, 
(a)

(c)

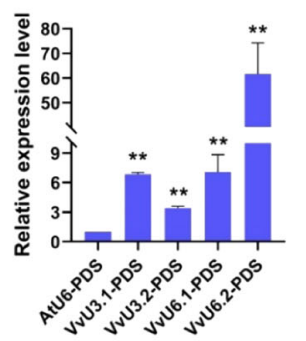

(b)
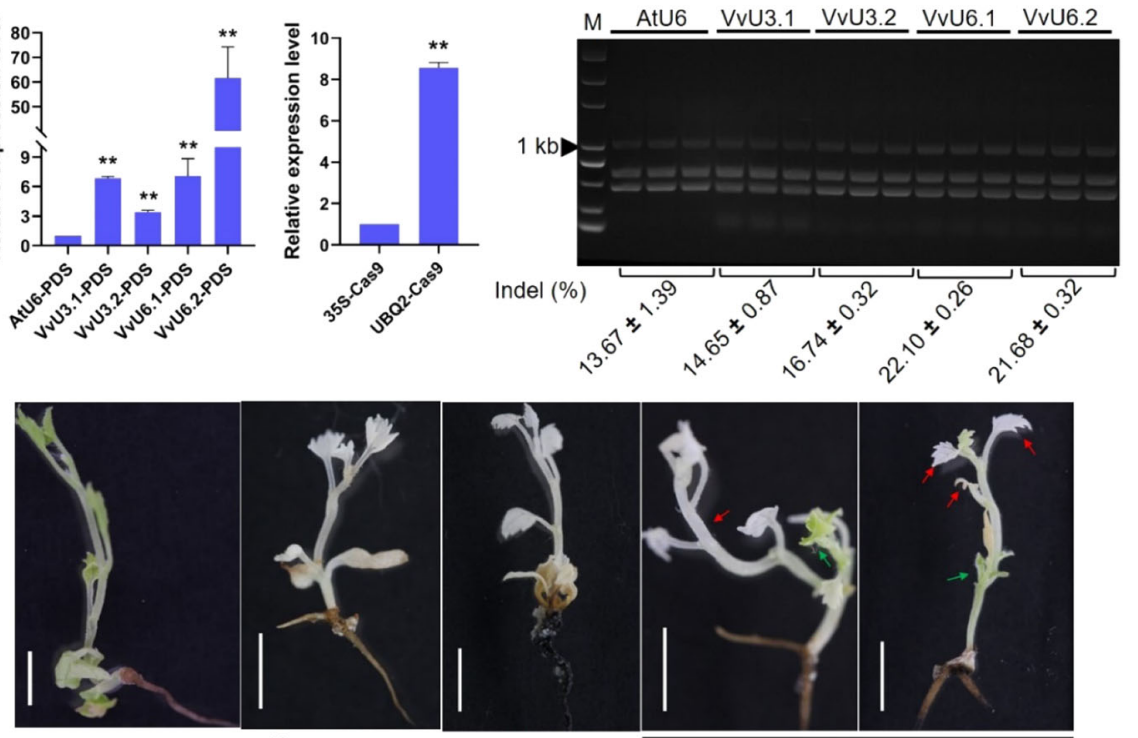

WT

Homozygous

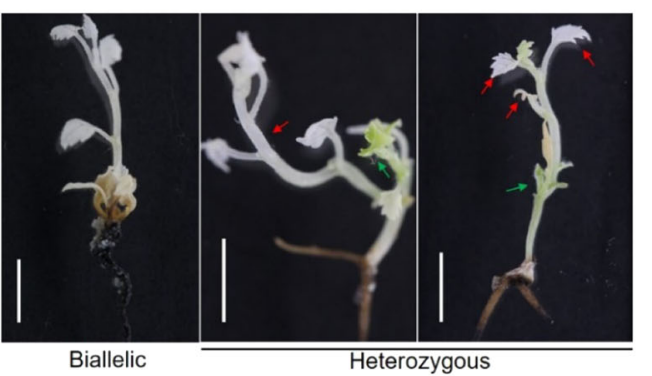

(d)

\begin{tabular}{lllll}
\hline & Mutation rate (\%) & $\begin{array}{l}\text { Homologous/Biallelic rate } \\
(\%)\end{array}$ & $\begin{array}{l}\text { Heterozygous rate } \\
(\%)\end{array}$ & $\begin{array}{l}\text { Wild-type rate } \\
(\%)\end{array}$ \\
\hline AtU6-PDS & $23.53(4 / 17)$ & $0(0 / 17)$ & $23.53(4 / 17)$ & $76.47(13 / 17)$ \\
VvU6.1-PDS & $34.48(10 / 29)$ & $13.79(4 / 29)$ & $20.69(6 / 29)$ & $65.52(19 / 29)$ \\
VvU6.2-PDS & $43.24(16 / 37)$ & $16.22(6 / 37)$ & $27.03(10 / 37)$ & $56.76(21 / 37)$ \\
\hline
\end{tabular}

Fig. 4 High-efficiency genome editing in transgenic grapevine plants. The most commonly used AtU6 and 355 promoters served as controls in the analysis of the efficiency of grape promoters. a Expression profiles of PDS sgRNA and Cas9 in 41B cells. The relative expression levels of sgRNA and Cas 9 were determined by qRT-PCR, and the AtU6-PDS and 35S-Cas9 constructs were used as controls. The data are shown as the mean values \pm SDs from three biological replicates; ${ }^{* *}$ indicates a highly significant $(P<0.01)$ difference. $\mathbf{b}$ Restriction fragment length polymorphism (RFLP) analysis of the editing efficiency. The PCR amplicons were treated with the Sspl enzyme and then separated on an EtBr-stained agarose gel (1.5\%). The digested bands are indicated by red triangles. The intensities of the bands were quantified with ImageJ. The intensity of an undigested band relative to the whole band was calculated as an indel mutation ${ }^{40}$. The data are shown as the mean values \pm SDs from three technical replicates. $\mathbf{c}$ Albino phenotypes of grapevine plants after targeted editing. Several albino plants are shown as examples. The albino parts of the heterozygous plants are indicated by red arrows and the green parts are denoted by green arrows. Scale bars: $0.5 \mathrm{~cm}$. $\mathbf{d}$ Overview of site-specific editing using the $U 6$ promoters in grapevine

we found that TMT1 and TMT2 exhibited high transcript levels in grape berries, particularly at veraison and during ripening, whereas the expression of TMT3 was poor or not detected in berries (Supplementary Figs. S6 and S7). Similar results were observed in 41B cells (Supplementary Fig. S8). These findings suggested that TMT1 and TMT2 play a vital role in sugar accumulation in vacuoles. Hence, we selected TMT1 and TMT2 as our target genes. Specific sgRNAs targeting TMT1 and TMT2 were designed and ligated to the VvU6.1 and VvU6.2 promoters, respectively. The two individual sgRNA expression cassettes were connected by overlapping PCR, and the combined multisgRNA expression cassette was used instead of the sgRNA expression cassette in the pCA-UBQ2-Cas9-VvU3/U6sgRNA vector to generate the multiplex editing vector pCA-UBQ2-Cas9-VvU6-TMTs (Fig. 5a). Furthermore, we synthesized the polycistronic tRNA-TMT sgRNA cassette
(Supplementary Fig. S9) and developed the PTG/ Cas9 system, in which the expression of Cas9, sgRNAs, and tRNA was driven by the single UBQ2 promoter (Fig. 5a).

The constructed vectors were introduced into 41B cells. After transformation and antibiotic-dependent selection, transgenic grape cells were recovered for mutation identification. Both the multi-sgRNA/Cas9 and PTG/ Cas9 systems could successfully induce the desired mutagenesis in the target genes. As shown in Fig. 5b, mutated sequences were detected in the amplicons of both the TMT1 and TMT2 target sequences. Single nucleotide insertions and nucleotide deletions were observed at the target sites (Fig. 5b), consistent with previous reports ${ }^{27,31,40}$. The T7EI assay revealed that the multi-sgRNA/Cas9 system resulted in editing efficiencies of $10.51 \%$ and $20.85 \%$ at the TMT1 and TMT2 target sites, respectively (Fig. 5c and Supplementary Table S2). 
(a)

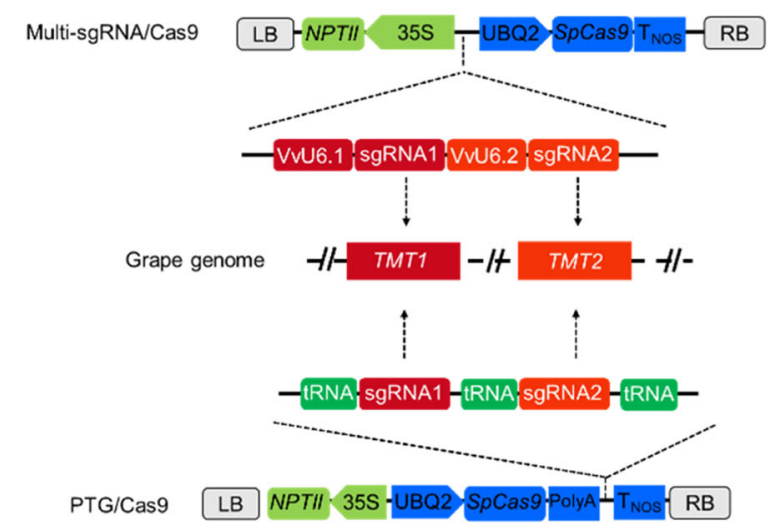

(b)

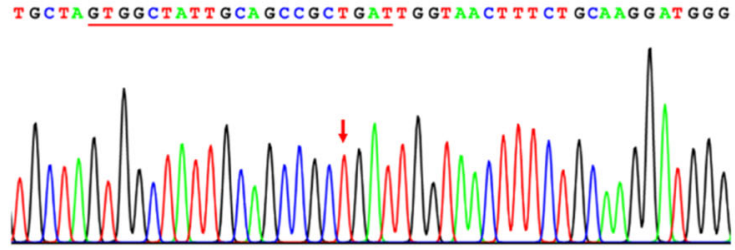

TMT1 target: GTGGCTATTGCAGCCGC. GATTGG

TTGCTAGTGGCTATTGCAGCCGCCGATTGGTAACTTTCTGCAAGGATGGG +1/C 3/10

Mut TGCTAGTGGCTATTGCAGCCGCT GATTGGTAACTTTCTGCAAGGATGGG $+1 /$ T $4 / 10$

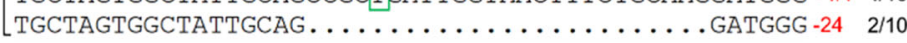

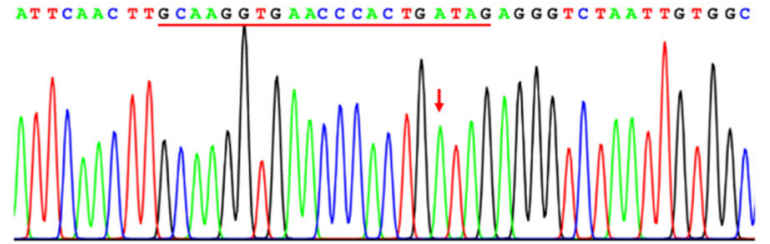

TMT2 target: GCAAGGTGAACCCACTG. TAGAGG

ATTCAACTTGCAAGGTGAACCCACTGA No.

ATTCAACTTGCAAGGTGAACCCACTGTTAGAGGGTCTAATTGTGGC $+1 / \pi \quad 4 / 10$

ATTCAACTTGCAAGGTGAACCCACT . . TAGAGGGTCTAATTGTGGC -1 $-1 / 10$

$\begin{array}{lll}\text { ATTCAACTTGCAAAGTGAACCC. . . . . TAGAGGGTCTAATTGTGGC } & -4 & 1 / 10\end{array}$

(c)

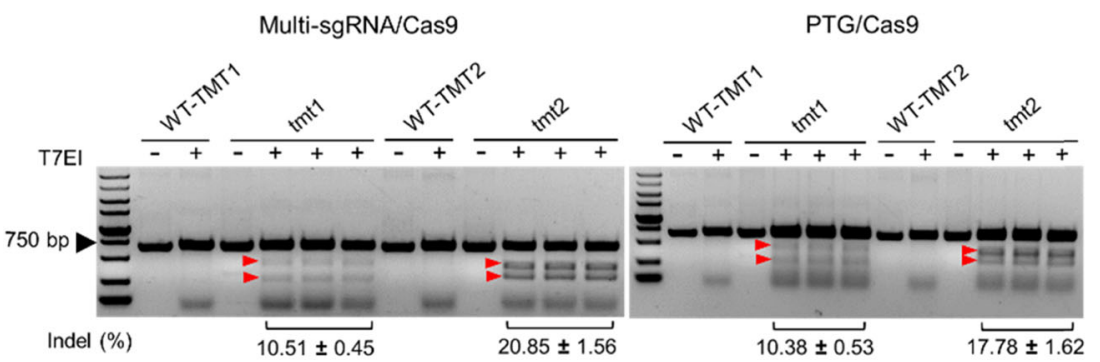

Fig. 5 Multiplex editing in grape using multi-sgRNA/Cas9 and PTG/Cas9 systems. a Schematic diagrams of the multi-sgRNA/Cas9 and PTG/ Cas 9 vectors and the editing targets. $\mathbf{b}$ Sanger sequencing results of the editing at TMT1 and TMT2 target sites. The gDNA was extracted from transformed $41 \mathrm{~B}$ cells for amplification of the target sequences. A total of 10 amplicons were sent for sequencing. A representative chromatogram and the mutated sequences identified for each target are shown. The target regions are underlined, and the mutation sites are indicated with red arrows in the chromatograms. The mutation types, as well as the corresponding number of sequences, are shown on the right. The inserted nucleotides are shown by green boxes. Mut, mutated sequences. c T7El assay of the editing efficiencies at TMT1 and TMT2 target sites. The PCR amplicons containing the TMT1 or TMT2 target sequences were treated with the T7El enzyme, and the digested products were separated on an EtBrstained agarose gel (1.5\%). The wild-type sequences of TMT1 (WT-TMT1) or TMT2 (WT-TMT2) served as controls. The intensities of the bands were quantified using ImageJ. The indel efficiencies calculated from the band intensities ${ }^{40}$ are shown as the mean values \pm SDs from three technical replicates; +, T7El digestion; -, no digestion 


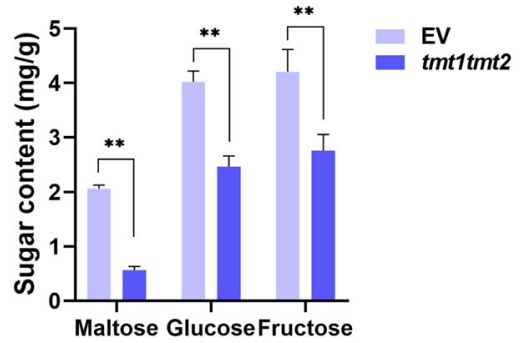

Fig. 6 Sugar content in the tmt1tmt 2 cells. Edited cells obtained using the multi-sgRNA/Cas9 system were collected as examples for determination of the sugar content, and grape cells transformed with empty vector (EV) were used as a control. The data are shown as the mean values \pm SDs from four biological replicates; ${ }^{* *}$ indicates a highly significant $(P<0.01)$ difference

Comparable mutation efficiencies were also generated using the PTG/Cas9 system at the TMT1 (10.38\%) and TMT2 (17.78\%) sites (Fig. 5c and Supplementary Table S2). Intriguingly, the TMT2 sgRNA had a higher editing efficiency than the TMT1 sgRNA in both the multisgRNA/Cas9 and PTG/Cas9 systems (Fig. 5c), which suggested that the sgRNA sequence had an effect on the editing efficiency. To evaluate the effect of TMT1 and TMT2 mutagenesis on sugar accumulation, we measured the sugar content in the edited $41 \mathrm{~B}$ cells. Compared with the cells transformed with empty vector (EV), the contents of maltose, glucose, and fructose in the tmt1tmt2 cells were significantly reduced (Fig. 6), which suggested that the mutation of TMT1 and TMT2 affected the extent of sugar accumulation in grape cells.

\section{The VvU3/U6 and UBQ2 promoters are suitable for genome editing in tobacco}

As mentioned above, the VvU3/VvU6 and UBQ2 promoters can drive the expression of sgRNA and GUS reporter genes in $N$. benthamiana leaves (Fig. 2a, b), which suggests that these promoters might be used for genome editing in tobacco. To confirm the efficacy of the VvU3/U6 and UBQ2 promoters in tobacco genome editing, the grape PDS sgRNA in the pCA-UBQ2-Cas9-VvU3/U6-PDS vectors (Fig. 2a) was replaced by the NbPDS sgRNA (Fig. 7a). The modified vectors were infiltrated into $N$. benthamiana leaves for transient expression. The tobacco leaves were sampled for mutation identification. Sanger sequencing assays showed that the use of the VvU3/U6 and UBQ2 promoters resulted in successful targeted mutagenesis at the target site in the NbPDS gene (Fig. 7b), which confirmed the efficacy of the grape Pol II and Pol III promoters in tobacco genome editing.

\section{Discussion}

As an RNA-guided DNA endonuclease system, sgRNA activity and the expression of sgRNA/Cas9 greatly

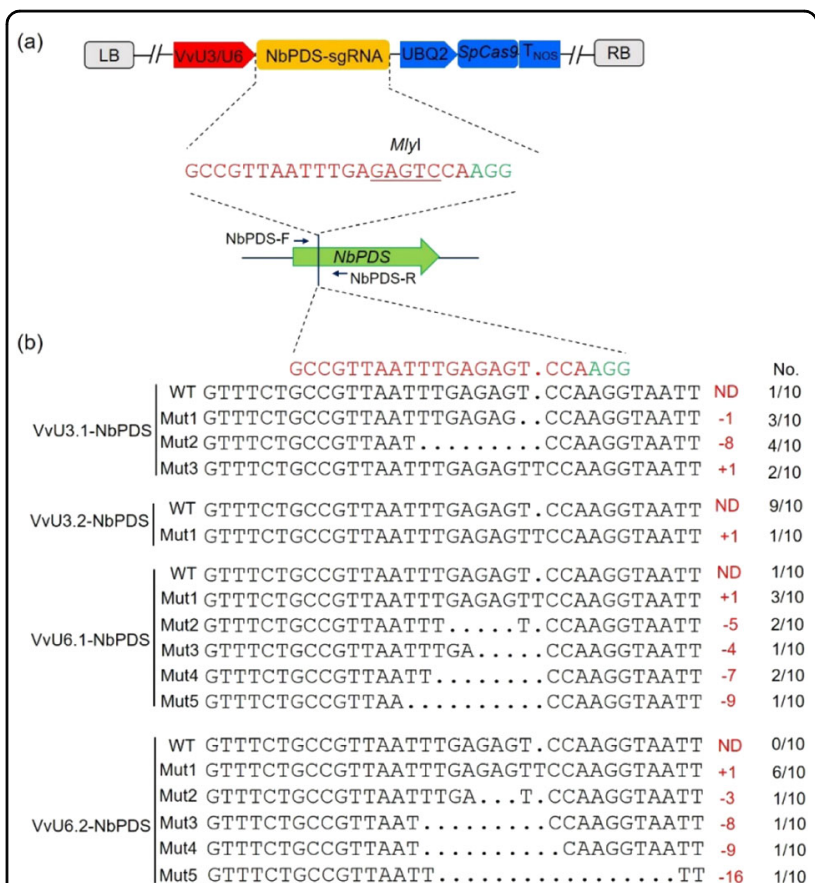

Fig. 7 Targeted editing of the NbPDS gene in tobacco leaves using grape promoters. a Schematic diagram of the CRISPR vectors and target selection for tobacco genome editing. The primers NbPDSF/R were used to amplify the target sequence of the NbPDS gene. The Mlyl restriction enzyme site is underlined. $\mathbf{b}$ Sequencing results of targeted mutations in the NbPDS. The CRISPR vectors harboring the VvU3 and VvU6 promoters were infiltrated into $N$. benthamiana leaves for transient expression. The mutated sequences (Mut) were analyzed by RE/PCR and Sanger sequencing assays. The mutation types and corresponding number of mutated sequences are shown in red and black, respectively, on the right

influence the efficiency of CRISPR/Cas9-mediated genome editing $^{47,48}$. The application of plant species-specific promoters is an effective strategy for improving the efficiency of genome engineering in plants ${ }^{8,10}$. To date, the widely used eukaryotic U3 and U6 promoters in monocot and dicot plants have been isolated from rice and Arabidopsis, respectively ${ }^{21,23}$. In grapevine, the AtU6 and $35 \mathrm{~S}$ promoters are commonly used to drive the expression of sgRNA and Cas9, respectively, in genome editing ${ }^{27,31,40}$. In this study, we characterized four Pol III promoters (VvU3.1, VvU3.2, VvU6.1, and VvU6.2) and one Pol II promoter (UBQ2) in grape and verified their efficiency in grape genome editing by targeting the PDS gene (Fig. 3). We established that both the UBQ2 and VvU3/U6 promoters exhibited strong activities in promoting the expression of Cas 9 and sgRNA in grape (Fig. 4a), which resulted in high editing efficiencies (Fig. 4b, d). The results demonstrated that the use of species-specific promoters could increase the genome editing efficiency in grape.

In addition, the amplified VvU3 and VvU6 promoters can be used for the expression of several distinct sgRNA 
modules, which can direct the Cas9 protein to edit different targets simultaneously. This Pol III promoterdependent strategy is commonly adopted for multiplex editing in plants ${ }^{13,15,16,21}$. In the present study, multiple sgRNA expression cassettes were assembled using the VvU6.1 and VvU6.2 promoters (Fig. 5a), because the first nucleotide of TMT1 and TMT2 sgRNAs is "G". This multi-sgRNA/Cas9 system was demonstrated to be effective in grape, with editing efficiencies of $10.51 \%$ and $20.85 \%$ at the TMT1 and TMT2 targets, respectively (Fig. 5c). Individual sgRNA expression cassettes are relatively easy to construct, and several assembly methods have been developed ${ }^{16,49}$. However, the use of many promoters results in a large expression vector, and size limitation remains a challenge when expressing an increased number of sgRNAs ${ }^{32}$. Plant Pol III promoters generally require conserved elements of the USE and TATA-like box for effective transcription, and shortened U3 and U6 promoters containing only the USE, TATA box, and several artificial monocot-specific promoter elements have been developed for genome editing in rice $^{21}$. Our identified VvU3 and VvU6 promoters contained the required USE and TATA-like box elements (Fig. 1), which suggested that the current promoters could also be optimized in the future. An alternative strategy for multiplex genome editing is expressing multiple sgRNAs from a single transcript ${ }^{34}$. In this case, endoribonuclease Csy4, tRNA, or RZs are generally employed to generate different sgRNAs ${ }^{34}$. However, Csy4 should be coexpressed with an artificial sgRNA array when using the Csy4-based sgRNA expression method ${ }^{34}$. Furthermore, in rice, the RZ system exhibited relatively poor editing activity compared with the tRNA system ${ }^{14}$. The tRNA-based PTG system is a very efficient approach for the simultaneous editing of several genes of interest with high efficiencies in plants $^{36,50}$. We also investigated the efficacy of the PTG/ Cas9 system in grape, and the results showed that the PTG/Cas9 system was as effective as the multi-sgRNA/ Cas9 system in editing the TMT1 and TMT2 genes (Fig. 5). The editing efficiencies using the PTG/ Cas9 system were slightly lower than those obtained using the multi-sgRNA/Cas9 system (Fig. 5c), and the difference could be explained by different promoters or processing efficiencies of the primary transcript.

To date, the CRISPR/Cas9 system has been a powerful tool for crop improvement. For instance, the simultaneous editing of three different genes helps increase the yield and cold tolerance of rice ${ }^{15}$, and the engineering of three homoeoalleles in bread wheat confers resistance to powdery mildew ${ }^{51}$. In grapevine, however, the CRISPR/ Cas9 system has not been fully exploited, and the application of this technology is restricted to single gene editing due to the limited CRISPR toolbox available. Multiple genes generally control the important traits involved in the quality of fruits of grape, such as sugar and organic acid accumulation ${ }^{46,52}$. TMTs are important sugar transporters that are reportedly associated with sugar accumulation in sugar beets and watermelon ${ }^{53,54}$. Of the three TMT genes in grape, TMT1 and TMT2 are thought to be involved in sugar accumulation in grape berries $^{46}$. Knockout of the TMT1 and TMT2 genes in grape cells significantly reduced the sugar content (Fig. 6). The simultaneous editing of the grape TMT1 and TMT2 genes provides evidence that can be used to deduce the function of these two genes in sugar accumulation in grape and constitutes, to our knowledge, the first example of multiplex genome editing in grape.

In conclusion, our study provides effective and robust grape Pol II and Pol III promoters for the optimization of the CRISPR/Cas9 system, and these promoters increase the editing efficiency in grape. Moreover, the developed multiplex genome editing systems, including the multisgRNA/Cas9 and PTG/Cas9 systems, expand the toolbox of grape genome editing and can thus facilitate the application of CRISPR/Cas9 technology to the future study of functional genes and mutant phenotypes in grape.

\section{Materials and methods}

\section{Plant materials and culture conditions}

Grapevine plants of $V$. vinifera cv. Pinot Noir were grown in the germplasm resources garden at the Institute of Botany, the Chinese Academy of Sciences, Beijing, under natural conditions. The leaves were sampled for gDNA isolation and the prepared gDNA was used for promoter cloning. 41B (Vitis vinifera $\mathrm{cv}$. Chasselas $\times$ Vitis berlandieri, a rootstock) cells were cultured in liquid glycerol-maltose (GM) medium as previously reported ${ }^{40}$. In vitro plants of Vitis amurensis were subcultured in half-strength Murashige \& Skoog medium (PhytoTech). Seeds of $N$. benthamiana were sown in the soil. Both the $V$. amurensis and tobacco plants grew at $26^{\circ} \mathrm{C}$ under long-day conditions (16-h light/8-h dark). Leaves of $V$. amurensis and tobacco plants were used for transient expression experiments.

\section{Cloning of promoters and construction of CRISPR vectors}

The Arabidopsis snRNAs AtU3b (AT5G53902) and AtU6-26 (AT3G13855) and the AtUBQ gene (AT3G52590) sequences were used as queries to identify the grape $U 3, U 6$, and $U B Q$ genes from the grape genome (http://plants.ensembl.org/Vitis_vinifera/Info/ Index), respectively. Approximately 500-800-bp DNA fragments upstream of the transcription start sites of the identified genes were amplified and cloned into the pLB vector (TIANGEN) for sequencing. The correct plasmids of VvU3.1-pLB, VvU3.2-pLB, VvU6.1-pLB, VvU6.2-pLB, UBQ1-pLB, and UBQ2-pLB were kept as templates for subsequent PCR. 
To develop the GUS reporter vectors, the UBQ1 and UBQ2 promoters were amplified from the $\mathrm{PLB}$ vector and cloned into the pBI121 vector instead of the $35 \mathrm{~S}$ promoter via the BamHI and HindIII sites through homologous recombination (HR) using the ClonExpress II One Step Cloning Kit (Vazyme). To inspect the efficacy of the VvU3 and VvU6 promoters in driving the expression of sgRNA, the grape PDS sgRNA was ligated to the VvU3 and VvU6 promoters by PCR, and the VvU3/U6-PDS expression cassettes were cloned into the $\mathrm{pCACRISPR/Cas9} \mathrm{vector} \mathrm{as}$ previously described ${ }^{27}$. To construct the pCA-UBQ2Cas9-VvU3/U6-PDS vectors, the Cas9 gene was amplified from the pCACRISPR/Cas9 vector and cloned into the HindIII-digested pCAMBIA2300 vector by HR. The UBQ2 promoter was then amplified and cloned into the pCAMBIA2300-Cas9 vector just upstream of the Cas9 gene via the BamHI and SalI sites through HR. The VvU3-PDS and VvU6-PDS expression cassettes were subsequently amplified from the well-constructed pCACRISPR/Cas9 vectors described above and ligated into the SmaI-digested pCAMBIA2300-UBQ2-Cas9 vector via HR. Similarly, to construct the CRISPR vectors for tobacco genome editing, the NbPDS sgRNA was fused to the VvU3 and VvU6 promoters by PCR, and the VvU3/ U6-NbPDS expression cassettes were introduced into the pCAMBIA2300-UBQ2-Cas9 vector by HR.

To construct the multi-sgRNA/Cas9 vector for multiplex genome editing, the TMT1 sgRNA and TMT2 sgRNA were fused to the VvU6.1 and VvU6.2 promoters by PCR, respectively, and the two sgRNA expression cassettes were combined by overlapping PCR. The combined sgRNA expression cassette was cloned into the SmaI-digested pCAMBIA2300-UBQ2-Cas9 vector via HR. For construction of the PTG/Cas9 vector, a DNA fragment containing a 50-bp polyA sequence and the polycistronic tRNA-TMT sgRNA expression cassette (Supplementary Fig. S6) was synthesized (Tsingke) and ligated into the HindIII-digested pCAMBIA2300-UBQ2-Cas9 vector by HR. All the primers used for cloning and vector construction are provided in Supplementary Table S1.

\section{Plant transformation, regeneration, and identification}

For the stable transformation of $41 \mathrm{~B}$ cells, the CRISPR vectors were introduced into the Agrobacterium strain EHA105, and Agrobacterium-mediated transformation of $41 \mathrm{~B}$ cells and the regeneration of transgenic plants were conducted as previously described ${ }^{31}$. After transformation, $41 \mathrm{~B}$ cells were cultured in liquid GM supplemented with $200 \mathrm{mg} / \mathrm{L}$ Timentin and $5 \mathrm{mg} / \mathrm{L}$ kanamycin, and the grape cells were subcultured every 4 weeks until the development of kanamycin-resistant cells. The resistant cells were sampled for gDNA isolation, and Cas9-specific primers (Supplementary Table S1) were used to identify T-DNA insertions. For the regeneration of transgenic plants, $41 \mathrm{~B}$ cells were transferred to GM without phytohormones. The induced embryos were further germinated on woody plant medium (Duchefa Biochemie) under light conditions $^{31}$. The regenerated plants were sampled for gDNA extraction, and the prepared gDNA was used for the PCR amplification of target fragments. The plants were analyzed by direct sequencing of PCR products followed by sequencing assays of individual amplicon clones $^{23}$. For each plant, a total of 25 clones were analyzed to verify the mutation types.

For transient expression experiments, the CRISPR vectors and GUS reporter vectors were introduced into Agrobacterium strain GV3101. The bacterial cells were cultured in liquid lysogeny broth medium containing $50 \mathrm{mg} / \mathrm{L}$ rifampicin and $50 \mathrm{mg} / \mathrm{L}$ kanamycin at $28^{\circ} \mathrm{C}$ overnight. The bacterial cultures were centrifuged at $5000 \times g$ for $10 \mathrm{~min}$, and the resultant supernatant was discarded, and the bacterial cells were resuspended in transfection buffer (10 $\mathrm{mM}$ MES, $10 \mathrm{mM} \mathrm{MgCl}_{2}$, and $200 \mu \mathrm{M}$ acetosyringone, $\mathrm{pH}$ 5.6). The concentration of the bacterial suspension was adjusted to a final $\mathrm{OD}_{600}$ of 0.4 . The suspension was incubated at room temperature for another $2 \mathrm{~h}$ before transformation. The leaves of 5 -weekold tobacco plants were infiltrated using a 1-mL needleless syringe. Three leaves from three independent plants were harvested as three biological replicates for qRT-PCR assay or GUS staining. For transient expression in grapevine leaves, the leaves of 1-month-old $V$. amurensis plants were immersed in the bacterial suspension, and vacuum was applied for 5-10 min until the leaves became translucent. After infiltration, the grapevine leaves were washed with sterile water and placed on sterile watersoaked filter paper in 90-mm Petri dishes. At least three independent $V$. amurensis plants were used for transformation with each vector. GUS staining of leaves was performed 3 days after infiltration.

\section{Mutation detection}

For the detection of mutagenesis in the target genes, DNA fragments containing the target sites were amplified by PCR with gene-specific primers from grape or tobacco. gDNA was first treated with the $S s p$ I enzyme to digest the wild-type sequence, and the target sequences of the grape PDS gene were then amplified. The PCR products were purified and cloned into the pLB vector for Sanger sequencing. For evaluation of the editing efficiencies, the RFLP method and T7EI assay were performed as previously described ${ }^{40}$. The PDS gene contained an SspI site within the target sequence, and digestion of the PCR amplicons with the restriction enzyme generated undigested bands. The indel frequency was measured based on the intensity of the undigested bands ${ }^{40}$. However, the target sequences of TMT1 and TMT2 lacked proper restriction enzyme sites; thus, the T7EI assay was adopted 
for assessment of the editing efficiencies. The editinginduced mismatched amplicons could be cleaved by the T7EI enzyme, and the indel frequency can be calculated from the intensities of the digested bands ${ }^{40}$. The PCR products treated with $S s p \mathrm{I}$ (NEB) or T7EI (NEB) enzyme were separated on an EtBr-stained agarose gel (1.5\%). The mutation efficiencies were determined using ImageJ.

\section{qRT-PCR assay}

Total RNA was extracted using the HiPure HP Plant RNA Mini Kit, and cDNA was synthesized from $1 \mu \mathrm{g}$ of total RNA using the HiScript II Q RT SuperMix for qPCR Kit (Vazyme) following the manufacturer's protocols. qRT-PCR assays were performed to determine the expression levels of the sgRNA and Cas9 genes in grape cells, and grape Actin 1 (accession no. AY680701) and GAPDH (accession no. XM 002263109) were used as internal controls. In tobacco leaves, the expression of sgRNA relative to that of the Cas9 gene was measured. The relative expression levels were calculated using the $2^{-\Delta \Delta C T}$ method $^{55}$. The significance of gene expression was determined using Student's $t$-test.

\section{Measurement of the sugar content}

The content of soluble sugars was determined using an HPLC system as described by Zhang et al. ${ }^{56}$.

\section{GUS staining}

GUS staining was performed as described by Baltes et al. ${ }^{57}$.

\section{Acknowledgements}

This work was jointly funded by grants from the National Natural Science Foundation of China (31772266 and 32001994) and the Bureau of International Cooperation of the Chinese Academy of Sciences (151111KYSB20170032).

\section{Author contributions}

C.R. and Z.L. conceived the study. C.R., Y.L., and Y.G. performed the experiments. W.D. and P.F. contributed to the experiments. C.R. wrote the manuscript. S.L. and Z.L. revised the manuscript. All of the authors approved the final manuscript.

\section{Conflict of interest}

The authors declare no competing interests.

Supplementary information The online version contains supplementary material available at https://doi.org/10.1038/s41438-021-00489-z.

Received: 17 September 2020 Revised: 22 December 2020 Accepted: 29 December 2020

Published online: 01 March 2021

\section{References}

1. Hsu, P. D., Lander, E. S. \& Zhang, F. Development and applications of CRISPRCas9 for genome engineering. Cell 157, 1262-1278 (2014).

2. Mackelprang, R. \& Lemaux, P. G. Genetic engineering and editing of plants: an analysis of new and persisting questions. Annu. Rev. Plant Biol. 71, 2.1-2.29 (2020).
3. Belhaj, K., Chrparro-Garcia, A., Kamoun, S., Patron, N. J. \& Nekrasov, V. Editing plant genomes with CRISPR/Cas9. Curr. Opin. Biotechnol. 32, 76-84 (2015).

4. Yin, K, Gao, C. \& Qiu, J. L. Progress and prospects in plant genome editing. Nat. Plants 3, 17107 (2017).

5. Malzahn, A., Lowder, L. \& Qi, Y. Plant genome editing with TALEN and CRISPR. Cell Biosci. 7, 21 (2017).

6. Jinek, $\mathrm{M}$. et al. A programmable dual-RNA-guided DNA endonuclease in adaptive bacterial immunity. Science 337, 816-821 (2012).

7. Jiang, W. et al. Demonstration of CRISPR/Cas9/sgRNA-mediated targeted gene modification in Arabidopsis, tobacco, sorghum and rice. Nucleic Acids Res. 41, e188 (2013).

8. Sun, X. et al. Targeted mutagenesis in soybean using the CRISPR-Cas9 system. Sci. Rep. 5, 10342 (2015)

9. Ng, H. \& Dean, N. Dramatic improvement of CRISPR/Cas9 editing in Candida albicans by increased single guide RNA expression. mSphere 2, e00385-16 (2017).

10. Long, L. et al. Optimization of CRISPR/Cas9 genome editing in cotton by improved sgRNA expression. Plant Methods 14, 85 (2018).

11. Nekrasov, V., Staskawicz, B., Weigel, D., Jones, J. D. \& Kamoun, S. Targeted mutagenesis in the model plant Nicotiana benthamiana using Cas9 RNAguided endonuclease. Nat. Biotechnol. 31, 691-693 (2013).

12. Mao, Y. et al. Application of the CRISPR-Cas system for efficient genome engineering in plants. Mol. Plant 6, 2008-2011 (2013).

13. Zhang, Z. et al. A multiplex CRISPR/Cas9 platform for fast and efficient editing of multiple genes in Arabidopsis. Plant Cell Rep. 35, 1519-1533 (2016).

14. Zhong, Z. et al. Intron-based single transcript unit CRISPR systems for plant genome editing. Rice 13, 8 (2020).

15. Zeng, Y., Wen, J., Zhao, W., Wang, Q. \& Huang, W. Rational improvement of rice yield and cold tolerance by editing the three genes OsPIN5b, GS3, and OsMYB30 with the CRISPR-Cas9 System. Front. Plant Sci. 10, 1663 (2020).

16. Lowder, L. G. et al. A CRISPR/Cas9 toolbox for multiplexed plant genome editing and transcriptional regulation. Plant Physiol. 169, 971-985 (2015).

17. Friedland, A. E. et al. Heritable genome editing in C elegans via a CRISPR-Cas9 system. Nat. Methods 10, 741-743 (2013).

18. Li, X., Jiang, D. H., Yong, K. \& Zhang, D. B. Varied transcriptional efficiencies of multiple Arabidopsis U6 small nuclear RNA genes. J. Integr. Plant Biol. 49, 222-229 (2007).

19. Venema, J., Vos, H. R., Faber, A. W., van Venrooij, W. J. \& Raué, H. A. Yeast Rrp9p is an evolutionarily conserved U3 snoRNP protein essential for early pre-rRNA processing cleavages and requires box C for its association. RNA 6, 1660-1671 (2000).

20. Marz, M. \& Stadler, P. F. Comparative analysis of eukaryotic U3 snoRNA. RNA Biol. 6, 503-507 (2009).

21. Hao, Y. et al. Shortened snRNA promoters for efficient CRISPR/Cas-based multiplex genome editing in monocot plants. Sci. China Life Sci. 63, 933-935 (2020).

22. Shockey, J. Gene editing in plants: assessing the variables through a simplified case study. Plant Mol. Biol. 103, 75-89 (2020).

23. $\mathrm{Ma}, \mathrm{X}$. et al. A robust CRISPR/Cas9 system for convenient, high-efficiency multiplex genome editing in monocot and dicot plants. Mol. Plant 8, 1274-1284 (2015).

24. Wang, P. et al. High efficient multisites genome editing in allotetraploid cotton (Gossypium hirsutum) using CRISPR/Cas9 system. Plant Biotechnol. J. 16, 137-150 (2018).

25. Charrier, A. et al. Efficient targeted mutagenesis in apple and first time edition of pear using the CRISPR-Cas9 System. Front. Plant Sci. 10, 40 (2019).

26. Liu, H. et al. Efficient induction of haploid plants in wheat by editing of TaMTL using an optimized Agrobacterium-mediated CRISPR system. J. Exp. Bot. 71, 1337-1349 (2020).

27. Ren, C. et al. CRISPR/Cas9-mediated efficient targeted mutagenesis in Chardonnay (Vitis vinifera L.). Sci. Rep. 6, 32289 (2016).

28. Nakajima, I. et al. CRISPR/Cas9-mediated targeted mutagenesis in grape. PLoS ONE 12, e0177966 (2017).

29. Wang, X. et al. CRISPR/Cas9-mediated efficient targeted mutagenesis in grape in the first generation. Plant Biotechnol. J. 16, 844-855 (2018).

30. Osakabe, Y. et al. CRISPR-Cas9-mediated genome editing in apple and grapevine. Nat. Protoc. 13, 2844-2863 (2018).

31. Ren, C. et al. Knockout of VVCCD8 gene in grapevine affects shoot branching. BMC Plant Biol. 20, 47 (2020). 
32. Minkenberg, B., Wheatley, M. \& Yang, Y. CRISPR/Cas9-enabled multiplex genome editing and its application. Prog. Mol. Biol. Transl. Sci. 149, 111-132 (2017).

33. He, Y. et al. Self-cleaving ribozymes enable the production of guide RNAs from unlimited choices of promoters for CRISPR/Cas9 mediated genome editing. J. Genet. Genomics 44, 469-472 (2017).

34. Tang, $X$. et al. Single transcript unit CRISPR 2.0 systems for robust Cas9 and Cas12a mediated plant genome editing. Plant Biotechnol. J. 17, 1431-1445 (2019).

35. Armario Najera, V., Twyman, R. M., Christou, P. \& Zhu, C. Applications of multiplex genome editing in higher plants. Curr. Opin. Biotechnol. 59, 93-102 (2019).

36. Xie, K., Minkenberg, B. \& Yang, Y. Boosting CRISPR/Cas9 multiplex editing capability with the endogenous tRNA-processing system. Proc. Natl Acad. Sci. USA 112, 3570-3575 (2015).

37. Li, T. et al. Domestication of wild tomato is accelerated by genome editing. Nat. Biotechnol. 36, 1160-1163 (2018).

38. Kwon, C. T. et al. Rapid customization of Solanaceae fruit crops for urban agriculture. Nat. Biotechnol. 38, 182-188 (2020).

39. Sunitha, S. \& Rock, C. D. CRISPR/Cas9-mediated targeted mutagenesis of TAS4 and MYBA7 loci in grapevine rootstock 101-14. Transgenic Res. 29, 355-367 (2020).

40. Ren, F. et al. Efficiency optimization of CRISPR/Cas9-mediated targeted mutagenesis in grape. Front. Plant Sci. 10, 612 (2019).

41. Shan, Q., Wang, Y., Li, J. \& Gao, C. Genome editing in rice and wheat using the CRISPR/Cas system. Nat. Protoc. 9, 2395-2410 (2014)

42. Kim, S., Bae, T., Hwang, J. \& Kim, J. S. Rescue of high-specificity Cas9 variants using sgRNAs with matched 5' nucleotides. Genome Biol. 18, 218 (2017).

43. Zhang, D. et al. Perfectly matched 20-nucleotide guide RNA sequences enable robust genome editing using high-fidelity SpCas9 nucleases. Genome Biol. 18, 191 (2017).

44. Zhang, P. et al. Multiplex CRISPR/Cas9-mediated metabolic engineering increases soya bean isoflavone content and resistance to soya bean mosaic virus. Plant Biotechnol. J. 18, 1384-1395 (2020).
45. Lecourieux, F., Lecourieux, D., Vignault, C. \& Delrot, S. A sugar-inducible protein kinase, VVSK1, regulates hexose transport and sugar accumulation in grapevine cells. Plant Physiol. 152, 1096-1106 (2010).

46. Afoufa-Bastien, D. et al. The Vitis vinifera sugar transporter gene family: phylogenetic overview and macroarray expression profiling. BMC Plant Biol. 10 245 (2010).

47. Doench, J. G. et al. Optimized sgRNA design to maximize activity and minimize off-target effects of CRISPR-Cas9. Nat. Biotechnol. 34, 184-191 (2016).

48. Doench, J. G. et al. Rational design of highly active sgRNAs for CRISPRCas9-mediated gene inactivation. Nat. Biotechnol. 32, 1262-1267 (2014).

49. Peterson, B. A. et al. Genome-wide assessment of efficiency and specificity in CRISPR/Cas9 mediated multiple site targeting in Arabidopsis. PLOS ONE 11, e0162169 (2016)

50. Qi, W. et al. High-efficiency CRISPR/Cas9 multiplex gene editing using the glycine tRNA-processing system-based strategy in maize. BMC Biotechnol. 16 58 (2016).

51. Wang, Y. et al. Simultaneous editing of three homoeoalleles in hexaploid bread wheat confers heritable resistance to powdery mildew. Nat. Biotechnol. 32, 947-952 (2014).

52. Kuhn, N. et al. Berry ripening: recently heard through the grapevine. J. Exp. Bot 65, 4543-4559 (2014)

53. Jung, B. et al. Identification of the transporter responsible for sucrose accumulation in sugar beet taproots. Nat. Plants 1, 14001 (2015).

54. Ren, Y. et al. A tonoplast sugar transporter underlies a sugar accumulation QTL in watermelon. Plant Physiol. 176, 836-850 (2018).

55. Livak, K. J. \& Schmittgen, T. D. Analysis of relative gene expression data using real-time quantitative $P C R$ and the $2^{-\Delta C T}$ method. Methods 25, 402-408 (2001).

56. Zhang, Z. et al. VVSWEET10 mediates sugar accumulation in grapes. Genes $\mathbf{1 0}$ 255 (2019).

57. Baltes, N. J., Gil-Humanes, J., Cermak, T., Atkins, P. A. \& Voytas, D. F. DNA replicons for plant genome engineering. Plant Cell 26, 151-163 (2014). 